\title{
NO-GO THEOREM FOR SAMPLING-BASED SIGNAL PROCESSING
}

\author{
Holger Boche* \\ Technische Universität München \\ Lehrstuhl für Theoretische Informationstechnik
}

\author{
Ullrich J. Mönich ${ }^{\dagger}$ \\ Massachusetts Institute of Technology
Research Laboratory of Electronics
}

\begin{abstract}
The approximation of linear time-invariant (LTI) systems by sampling series is an important topic in signal processing. However, the convergence of the approximation process is not guaranteed. In this paper we prove that for every sampling pattern that is a complete interpolating sequence there exists a universal stable LTI system such that for every oversampling factor there exists a bandlimited input signal such that the approximation process, which is used to approximate the output signal of the LTI system, diverges. This result shows a fundamental limit for the digital sampling-based implementation of systems.
\end{abstract}

Index Terms - bandlimited signal, linear time-invariant system, complete interpolating sequence, approximation, divergence

\section{INTRODUCTION}

Sampling theory plays a fundamental role in modern signal and information processing, because it is the basis for today's digital world. In his seminal work [1] Shannon started this theory. The reconstruction of bandlimited signals from their samples is also widely used in other applications and theoretical concepts [2, 3, 4, 5]. For an overview of existing sampling theorems see for example $[2,6]$, and [7].

Although the sampling theorems are very important on their own, they do not reflect the actual purpose of signal processing. The core task of signal processing is to process data. This means that, usually, the interest is not in a reconstruction of the sampled signal itself, but in some processed version of it. This might be the derivative, the Hilbert transform or the output of any other stable linear system $T$. In the general case the goal is to approximate the desired transform $T f$ of a signal $f$ by an approximation process, which uses only finitely many, not necessarily equidistant, samples of the signal $f$. Exactly as in the case of signal reconstruction, the convergence and approximation behavior is important for practical applications [8]. form

In this paper we analyze system approximation processes of the

$$
\sum_{k=-\infty}^{\infty} f\left(t_{k}\right)\left(T \phi_{k}\right)(t)
$$

*This work was partly supported by the German Research Foundation (DFG) under grant BO 1734/22-1.

$\dagger$ U. Mönich was supported by the German Research Foundation (DFG) under grant MO 2572/1-1.

An extended version of this paper will be published as part of the book "New Perspectives on Approximation and Sampling Theory - Festschrift in honor of Paul Butzer's 85th birthday" in the Applied and Numerical Harmonic Analysis Series, Birkhäuser (Springer-Verlag). for signals $f$ in $\mathcal{P} \mathcal{W}_{\pi}^{1}$, i.e., the space of bandlimited signals with absolutely integrable Fourier transform. $\left\{t_{k}\right\}_{k \in \mathbb{Z}}$ denotes the sequence of sampling points. For the special case of equidistant sampling, (1) reduces to

$$
\frac{1}{a} \sum_{k=-\infty}^{\infty} f\left(\frac{k}{a}\right) h_{T}\left(t-\frac{k}{a}\right),
$$

where $a \geq 1$ denotes the oversampling factor and $h_{T}$ is the impulse response of the system $T$. It has already been shown that the Hilbert transform is a universal system for which there exists for every amount of oversampling a signal such that the peak value of (2) diverges [9]. Here we analyze whether the additional degree of freedom that is introduced by the non-equidistant sampling can be used to circumvent this divergence.

In [10] it was conjectured that (1) is not always stable, i.e, that there exist stable LTI systems $T$ and bandlimited signals $f$ such that (1) diverges, regardless of oversampling. Our result in Section 4 proves this conjecture.

\section{NOTATION}

Let $\hat{f}$ denote the Fourier transform of a function $f$, where $\hat{f}$ is to be understood in the distributional sense. $L^{p}(\mathbb{R}), 1 \leq p<\infty$, is the space of all to the $p$ th power Lebesgue integrable functions on $\mathbb{R}$, with the usual norm $\|\cdot\|_{p}$, and $L^{\infty}(\mathbb{R})$ the space of all functions for which the essential supremum norm $\|\cdot\|_{\infty}$ is finite. $C[a, b]$ denotes the space of all continuous functions on $[a, b]$. Further, $l^{p}, 1 \leq p<$ $\infty$, is the space of all sequences that are summable to the $p$ th power.

For $-\infty<\sigma_{1}<\sigma_{2}<\infty$ and $1 \leq p \leq \infty$ we denote by $\mathcal{P} \mathcal{W}_{\left[\sigma_{1}, \sigma_{2}\right]}^{p}$ the Paley-Wiener space of functions $f$ with a representation $f(z)=1 /(2 \pi) \int_{-\sigma_{1}}^{\sigma_{2}} g(\omega) \mathrm{e}^{i z \omega} \mathrm{d} \omega, z \in \mathbb{C}$, for some $g \in L^{p}\left[\sigma_{1}, \sigma_{2}\right]$. The norm for $\mathcal{P} \mathcal{W}_{\left[\sigma_{1}, \sigma_{2}\right]}^{p}, 1 \leq p<\infty$, is given by $\|f\|_{\mathcal{P} \mathcal{W}_{\left[\sigma_{1}, \sigma_{2}\right]}^{p}}=\left(1 /(2 \pi) \int_{\sigma_{1}}^{\sigma_{2}}|\hat{f}(\omega)|^{p} \mathrm{~d} \omega\right)^{1 / p}$. For $\mathcal{P} \mathcal{W}_{[-\sigma, \sigma]}^{p}$, $0<\sigma<\infty$, we use the abbreviation $\mathcal{P} \mathcal{W}_{\sigma}^{p}$.

We briefly review some definitions and facts about stable linear time-invariant (LTI) systems. A linear system $T: \mathcal{P} \mathcal{W}_{\pi}^{p} \rightarrow \mathcal{P} \mathcal{W}_{\pi}^{p}$, $1 \leq p \leq \infty$, is called stable if the operator $T$ is bounded, i.e., if $\|T\|:=\sup _{\|f\|_{\mathcal{P} \mathcal{W}^{p} \leq 1}}\|T f\|_{\mathcal{P} \mathcal{W}_{\pi}^{p}}<\infty$. Furthermore, it is called time-invariant if $(T f(\cdot-a))(t)=(T f)(t-a)$ for all $f \in \mathcal{P} \mathcal{W}_{\pi}^{p}$ and $t, a \in \mathbb{R}$. For every stable LTI system $T: \mathcal{P} \mathcal{W}_{\pi}^{1} \rightarrow \mathcal{P} \mathcal{W}_{\pi}^{1}$ there exists exactly one function $\hat{h}_{T} \in L^{\infty}[-\pi, \pi]$ such that

$$
(T f)(t)=\frac{1}{2 \pi} \int_{-\pi}^{\pi} \hat{f}(\omega) \hat{h}_{T}(\omega) \mathrm{e}^{i \omega t} \mathrm{~d} \omega, \quad t \in \mathbb{R},
$$

for all $f \in \mathcal{P} \mathcal{W}_{\pi}^{1}$. Conversely, every function $\hat{h}_{T} \in L^{\infty}[-\pi, \pi]$ defines a stable LTI system $T: \mathcal{P} \mathcal{W}_{\pi}^{1} \rightarrow \mathcal{P} \mathcal{W}_{\pi}^{1}$. The operator norm of a stable LTI system $T$ is given by $\|T\|=\|\hat{h}\|_{L^{\infty}[-\pi, \pi]}$. Note that $\hat{h}_{T} \in L^{\infty}[-\pi, \pi] \subset L^{2}[-\pi, \pi]$, and consequently $h_{T} \in \mathcal{P} \mathcal{W}_{\pi}^{2}$. 


\section{BASICS OF NON-EQUIDISTANT SAMPLING}

In classical non-equidistant sampling, the goal is to reconstruct a bandlimited signal $f$ from its non-equidistant samples $\left\{f\left(t_{k}\right)\right\}_{k \in \mathbb{Z}}$, where $\left\{t_{k}\right\}_{k \in \mathbb{Z}}$ is the sequence of sampling points. One possibility to do the reconstruction is to use the sampling series

$$
\sum_{k=-\infty}^{\infty} f\left(t_{k}\right) \phi_{k}(t)
$$

where the $\phi_{k}, k \in \mathbb{Z}$, are certain reconstruction functions, to be specified later.

In this paper we consider sampling point sequences $\left\{t_{k}\right\}_{k \in \mathbb{Z}}$ that are real and a complete interpolating sequence for $\mathcal{P} \mathcal{W}_{\pi}^{2}$.

Definition 1. We say that $\left\{t_{k}\right\}_{k \in \mathbb{Z}}$ is a complete interpolating sequence for $\mathcal{P} \mathcal{W}_{\pi}^{2}$ if the interpolation problem $f\left(t_{k}\right)=c_{k}, k \in \mathbb{Z}$, has exactly one solution $f \in \mathcal{P} \mathcal{W}_{\pi}^{2}$ for every sequence $\left\{c_{k}\right\}_{k \in \mathbb{Z}} \in$ $l^{2}$.

We further assume that the sequence of sampling points $\left\{t_{k}\right\}_{k \in \mathbb{Z}}$ is ordered strictly increasingly, and, without loss of generality, we assume that $t_{0}=0$. Then, it follows that the product

$$
\phi(z)=z \lim _{N \rightarrow \infty} \prod_{\substack{k \mid \leq N \\ k \neq 0}}\left(1-\frac{z}{t_{k}}\right)
$$

converges uniformly on $|z| \leq R$ for all $R<\infty$, and $\phi$ is an entire function of exponential type $\pi$ [11]. It can be seen from (5) that $\phi$, which is often called generating function, has the zeros $\left\{t_{k}\right\}_{k \in \mathbb{Z}}$. Moreover, it follows that

$$
\phi_{k}(t)=\frac{\phi(t)}{\phi^{\prime}\left(t_{k}\right)\left(t-t_{k}\right)}
$$

is the unique function in $\mathcal{P} \mathcal{W}_{\pi}^{2}$ that solves the interpolation problem $\phi_{k}\left(t_{l}\right)=\delta_{k l}$, where $\delta_{k l}=1$ if $k=l$, and $\delta_{k l}=0$ otherwise.

Remark 1. Equidistant sampling with $t_{k}=k, k \in \mathbb{Z}$, is a special case of the more general non-equidistant setting that is considered in this paper. For equidistant sampling we have $\phi_{k}(t)=\operatorname{sinc}(t-k)$, $k \in \mathbb{Z}$, and (4) reduces to the ordinary Shannon sampling series.

We also need the concept of a Riesz basis.

Definition 2. A system of vectors $\left\{\phi_{k}\right\}_{k \in \mathbb{Z}}$ in a separable Hilbert space $\mathcal{H}$ is called Riesz basis if $\left\{\phi_{k}\right\}_{k \in \mathbb{Z}}$ is complete in $\mathcal{H}$, and there exist positive constants $A$ and $B$ such that for all $M, N \in \mathbb{N}$ and arbitrary scalars $c_{k}$ we have

$$
A \sum_{k=-M}^{N}\left|c_{k}\right|^{2} \leq\left\|\sum_{k=-M}^{N} c_{k} \phi_{k}\right\|^{2} \leq B \sum_{k=-M}^{N}\left|c_{k}\right|^{2} .
$$

It is well-known [12, p. 143] that the system $\left\{\mathrm{e}^{i \omega t_{k}}\right\}_{k \in \mathbb{Z}}$ is a Riesz basis for $L^{2}[-\pi, \pi]$ if and only if $\left\{t_{k}\right\}_{k \in \mathbb{Z}}$ is a complete interpolating sequence for $\mathcal{P} \mathcal{W}_{\pi}^{2}$. From this fact it follows immediately that $\left\{\phi_{k}\right\}_{k \in \mathbb{Z}}$, as defined in (6), is a Riesz basis for $\mathcal{P} \mathcal{W}_{\pi}^{2}$ if $\left\{t_{k}\right\}_{k \in \mathbb{Z}}$ is a complete interpolating sequence for $\mathcal{P} \mathcal{W}_{\pi}^{2}$.

\section{NO-GO RESULT FOR SAMPLING-BASED SIGNAL APPROXIMATION}

Next, we treat the system approximation problem and analyze system approximation process (1).

Theorem 1. Let $\left\{t_{k}\right\}_{k \in \mathbb{Z}} \subset \mathbb{R}$ be an ordered complete interpolating sequence for $\mathcal{P} \mathcal{W}_{\pi}^{2}, \phi_{k}$ as defined in (6), and $t \in \mathbb{R}$. Then there exists a stable LTI system $T_{*}: \mathcal{P} \mathcal{W}_{\pi}^{1} \rightarrow \mathcal{P} \mathcal{W}_{\pi}^{1}$ such that for every $0<\sigma<\pi$ there exists a signal $f_{*} \in \mathcal{P} \mathcal{W}_{\sigma}^{1}$ such that

$$
\limsup _{N \rightarrow \infty}\left|\sum_{k=-N}^{N} f_{*}\left(t_{k}\right)\left(T_{*} \phi_{k}\right)(t)\right|=\infty
$$

Theorem 1 shows that a sampling-based implementation of stable LTI systems is not always possible for the signal space $\mathcal{P} \mathcal{W}_{\pi}^{1}$. This result illustrates the limits of a general sampling-based technology.

It is interesting to note that the system $T_{*}$ in Theorem 1 is universal in the sense that it does not depend on $\sigma$, i.e., the amount of oversampling. In other words, we can find a stable LTI system $T_{*}$ such that regardless of the oversampling factor $1<\alpha<\infty$ there exists a signal $f_{*} \in \mathcal{P} \mathcal{W}_{\pi / \alpha}^{1}$ for which the system approximation process diverges as in (8).

Theorem 1 is an abstract existence result, which states the existence of a stable LTI system $T_{*}$ and a signal $f_{*}$. However, we would like to mention that, using a technique from [9], it is possible to explicitly construct a divergence creating system $T_{*}$ and signal $f_{*}$.

Here we analyzed the behavior of the system approximation process for fixed $t \in \mathbb{R}$. If instead the peak value is considered, it was shown in [9] that, for the special case of equidistant sampling, the Hilbert transform is a divergence creating system.

Remark 2. Since $\left\{\phi_{k}\right\}_{k \in \mathbb{Z}}$ is a Riesz basis for $\mathcal{P} \mathcal{W}_{\pi}^{2}$, it follows that the projections of $\left\{\phi_{k}\right\}_{k \in \mathbb{Z}}$ onto $\mathcal{P} \mathcal{W}_{\sigma}^{2}$ form a frame for $\mathcal{P} \mathcal{W}_{\sigma}^{2}$, $0<\sigma<\pi$ [13, p. 231]. However, even though the projections of $\left\{\phi_{k}\right\}_{k \in \mathbb{Z}}$ onto $\mathcal{P} \mathcal{W}_{\sigma}^{2}$ form a frame for $\mathcal{P} \mathcal{W}_{\sigma}^{2}, 0<\sigma<\pi$, we have the divergence that was observed in Theorem 1.

The result of Theorem 1 is also true for bandpass signals. However, in this case the stable LTI system $T_{*}$ is no longer universal but depends on the actual frequency support of the signal space, as shown by the next theorem.

Theorem 2. Let $\left\{t_{k}\right\}_{k \in \mathbb{Z}} \subset \mathbb{R}$ be an ordered complete interpolating sequence for $\mathcal{P} \mathcal{W}_{\pi}^{2}$, $\phi_{k}$ as defined in (6), $t \in \mathbb{R}$, and $0<\sigma_{1}<\sigma_{2}<$ $\pi$. Then there exist a stable LTI system $T_{*}: \mathcal{P W}_{\pi}^{1} \rightarrow \mathcal{P} \mathcal{W}_{\pi}^{1}$ and a signal $f_{*} \in \mathcal{P} \mathcal{W}_{\left[\sigma_{1}, \sigma_{2}\right]}^{1}$ such that

$$
\limsup _{N \rightarrow \infty}\left|\sum_{k=-N}^{N} f_{*}\left(t_{k}\right)\left(T_{*} \phi_{k}\right)(t)\right|=\infty
$$

For the proofs of Theorems 1 and 2, we need two lemmas, Lemma 1 and Lemma 4.

Lemma 1. Let $\left\{t_{k}\right\}_{k \in \mathbb{Z}} \subset \mathbb{R}$ be an ordered complete interpolating sequence for $\mathcal{P} \mathcal{W}_{\pi}^{2}$ and $\phi_{k}$ as defined in (6). Then there exists a positive constant $C_{1}$ such that for all $\omega \in[-\pi, \pi]$ and all $N \in \mathbb{N}$ we have

$$
\max _{1 \leq M \leq N} \frac{1}{2 \pi} \int_{-\pi}^{\pi}\left|\sum_{k=-M}^{M} \mathrm{e}^{i \omega t_{k}} \hat{\phi}_{k}\left(\omega_{1}\right)\right| \mathrm{d} \omega_{1} \geq C_{1} \log (N) .
$$


For the proof of Lemma 1 we need Lemmas 2 and 3 from Szarek's paper [14]. For completeness and convenience, we state them next in a slightly simplified version, which is sufficient for our purposes.

Lemma 2 (Szarek). Let $f$ be a nonnegative measurable function, $\mathrm{C}_{2}$ a positive constant, and $n$ a natural number such that

$$
\frac{1}{2 \pi} \int_{-\pi}^{\pi}(f(t))^{2} \mathrm{~d} t \leq C_{2} n
$$$$
\text { and } \quad \frac{1}{2 \pi} \int_{-\pi}^{\pi}(f(t))^{5 / 4} \mathrm{~d} t \geq \frac{n^{1 / 4}}{C_{2}} .
$$

Then there exists a number $\alpha=\alpha\left(C_{2}\right), 0<\alpha<2^{-3}$ and a natural number s such that

$$
\begin{aligned}
\frac{1}{2 \pi} \int_{\left\{t \in[-\pi, \pi]: f(t)>\frac{n}{\alpha^{2}}\right\}} f(t) \mathrm{d} t \leq \frac{\alpha}{2^{4}} \\
\text { and } \quad \frac{1}{2 \pi} \int_{\left\{t \in[-\pi, \pi]: \frac{\alpha^{s} n}{\alpha^{2}}<f(t) \leq \frac{\alpha^{s} n}{\alpha^{3}}\right\}} f(t) \mathrm{d} t \geq s \alpha .
\end{aligned}
$$

Lemma 3 (Szarek). Let $0<\alpha<2^{-3}$ and $\left\{F_{k}\right\}_{k=1}^{N}$ be a sequence of measurable functions. Further, define $F_{k, n}:=F_{k+n}-F_{k}$. Assume that for all $k, n$ satisfying $1 \leq k, n$ and $1 \leq k+n \leq N$ there exists a natural number $s=s(k, n)$ such that

$$
\frac{1}{2 \pi} \int_{\left\{t \in[-\pi, \pi]:\left|F_{k, n}(t)\right|>\frac{n}{\alpha^{2}}\right\}}\left|F_{k, n}(t)\right| \mathrm{d} t \leq \frac{\alpha}{2^{4}}
$$

and $\quad \frac{1}{2 \pi} \int_{\left\{t \in[-\pi, \pi]: \frac{\alpha^{s} n}{\alpha^{2}}<\left|F_{k, n}(t)\right| \leq \frac{\alpha^{s} n}{\alpha^{3}}\right\}}\left|F_{k, n}(t)\right| \mathrm{d} t \geq s \alpha$.

Then there exists a positive constant $C_{3}=C_{3}(\alpha)$ such that

$$
\max _{1 \leq k \leq N} \frac{1}{2 \pi} \int_{-\pi}^{\pi}\left|F_{k}(t)\right| \mathrm{d} t \geq C_{3}(\alpha) \log (N) .
$$

Now we are in the position to prove Lemma 1.

Proof of Lemma 1. Let $\left\{t_{k}\right\}_{k \in \mathbb{Z}} \subset \mathbb{R}$ be an arbitrary but fixed ordered complete interpolating sequence for $\mathcal{P} \mathcal{W}_{\pi}^{2}$ and $\phi_{k}$ as defined in (6). Further, let $\omega \in[-\pi, \pi]$ be arbitrary but fixed. For $\omega_{1} \in$ $[-\pi, \pi]$ consider the functions $G_{k}\left(\omega_{1}, \omega\right):=\sum_{l=-k}^{k} \mathrm{e}^{i \omega t_{l}} \hat{\phi}_{l}\left(\omega_{1}\right)$, and

$$
\begin{aligned}
G_{k, n}\left(\omega_{1}, \omega\right) & :=G_{k+n}\left(\omega_{1}, \omega\right)-G_{k}\left(\omega_{1}, \omega\right) \\
& =\sum_{k<|l| \leq k+n} \mathrm{e}^{i \omega t_{l}} \hat{\phi}_{l}\left(\omega_{1}\right) .
\end{aligned}
$$

We will show that $\left|G_{k, n}\left(\omega_{1}, \omega\right)\right|$ satisfies the conditions (10) and (11) of Lemma 2. We have

$$
\begin{aligned}
\frac{1}{2 \pi} \int_{-\pi}^{\pi}\left|G_{k, n}\left(\omega_{1}, \omega\right)\right|^{2} \mathrm{~d} \omega_{1} & =\int_{-\infty}^{\infty}\left|\sum_{k<|l| \leq k+n} \mathrm{e}^{i \omega t_{l}} \phi_{l}(t)\right|^{2} \mathrm{~d} t \\
& \leq B \sum_{k<|l| \leq k+n} 1=B 2 n
\end{aligned}
$$

where we used the fact that $\left\{\phi_{l}\right\}_{k \in \mathbb{Z}}$ is a Riesz basis for $\mathcal{P} \mathcal{W}_{\pi}^{2}$. Next, we analyze the expression

$$
\frac{1}{2 \pi} \int_{-\pi}^{\pi}\left|G_{k}\left(\omega_{1}, \omega\right)\right|^{p} \mathrm{~d} \omega_{1}=\frac{1}{2 \pi} \int_{-\pi}^{\pi}\left|\sum_{l=-k}^{k} \mathrm{e}^{i \omega t_{l}} \hat{\phi}_{l}\left(\omega_{1}\right)\right|^{p} \mathrm{~d} \omega_{1}
$$

for $1<p<2$. We set $G_{k, n}\left(\omega_{1}, \omega\right)=0$ for $\left|\omega_{1}\right|>\pi$ and consider the Fourier transform

$$
\left(\mathcal{F} G_{k, n}(\cdot, \omega)\right)(t)=\int_{-\infty}^{\infty} G_{k, n}\left(\omega_{1}, \omega\right) \mathrm{e}^{-i \omega_{1} t} \mathrm{~d} \omega_{1} .
$$

We have $\left(\mathcal{F} G_{k, n}(\cdot, \omega)\right)(t)=2 \pi g_{k, n}(-t, \omega)$, where $g_{k, n}(t, \omega):=$ $\sum_{k<|l| \leq k+n} \mathrm{e}^{i \omega t_{l}} \phi_{l}(t)$. Let $q$ be the conjugate of $p$, i.e., $1 / p+$ $1 / q=1$, then the Hausdorff-Young inequality [15],[6, p. 19] shows that there exists a constant $C_{4}=C_{4}(p)$ such that

$$
\frac{1}{2 \pi} \int_{-\pi}^{\pi}\left|G_{k, n}\left(\omega_{1}, \omega\right)\right|^{p} \mathrm{~d} \omega_{1} \geq \frac{(2 \pi)^{p-1}}{\left(C_{4}(p)\right)^{p}}\left(\int_{-\infty}^{\infty}\left|g_{k, n}(t, \omega)\right|^{q} \mathrm{~d} t\right)^{\frac{p}{q}} .
$$

Note that the constant $C_{4}(p)$ is independent of $\omega$. We analyze the integral on the right-hand side of (13). We have $g_{k, n}(\cdot, \omega) \in \mathcal{B}_{\pi}^{q}$. Since $\left\{t_{k}\right\}_{k \in \mathbb{Z}}$ is a complete interpolating sequence for $\mathcal{P} \mathcal{W}_{\pi}^{2}$, we have [16] $\inf _{k \in \mathbb{Z}}\left(t_{k+1}-t_{k}\right)>0$, and it is known [17, p. 101] that there exists a positive constant $C_{5}(q)$ that is independent of $k, n$, and $\omega$ such that

$$
\left(\int_{-\infty}^{\infty}\left|g_{k, n}(t, \omega)\right|^{q} \mathrm{~d} t\right)^{\frac{1}{q}} \geq C_{5}(q)\left(\sum_{l=-\infty}^{\infty}\left|g_{k, n}\left(t_{l}, \omega\right)\right|^{q}\right)^{\frac{1}{q}} .
$$

Since $\sum_{l=-\infty}^{\infty}\left|g_{k, n}\left(t_{l}, \omega\right)\right|^{q}=\sum_{k<|l| \leq n+k} 1=2 n$, we obtain

$$
\left(\int_{-\infty}^{\infty}\left|g_{k, n}(t, \omega)\right|^{q} \mathrm{~d} t\right)^{\frac{1}{q}} \geq C_{5}(q)(2 n)^{\frac{1}{q}}
$$

Combining (13) and (14) gives

$$
\frac{1}{2 \pi} \int_{-\pi}^{\pi}\left|G_{k, n}\left(\omega_{1}, \omega\right)\right|^{p} \mathrm{~d} \omega_{1} \geq \frac{(2 \pi)^{p-1}\left(C_{5}(q)\right)^{p}}{\left(C_{4}(p)\right)^{p}}(2 n)^{\frac{p}{q}},
$$

and for $p=5 / 4$ we obtain

$$
\frac{1}{2 \pi} \int_{-\pi}^{\pi}\left|G_{k, n}\left(\omega_{1}, \omega\right)\right|^{\frac{5}{4}} \mathrm{~d} \omega_{1} \geq \frac{(4 \pi)^{\frac{1}{4}}\left(C_{5}(5)\right)^{\frac{5}{4}}}{\left(C_{4}\left(\frac{5}{4}\right)\right)^{\frac{5}{4}}} n^{\frac{1}{4}} .
$$

Choosing $C_{2}=\max \left\{2 B^{2},\left(C_{4}\left(\frac{5}{4}\right)\right)^{\frac{5}{4}} /\left((4 \pi)^{\frac{1}{4}}\left(C_{5}(5)\right)^{\frac{5}{4}}\right)\right\}$, we see from (12) and (15) that the function $\left|G_{k, n}\left(\omega_{1}, \omega\right)\right|$ satisfies conditions (10) and (11), that is the assumptions of Lemma 2. Hence, as a result of Lemma $2,\left|G_{k}\left(\omega_{1}, \omega\right)\right|$ also satisfies the assumptions of Lemma 3, and application of Lemma 3 completes the proof.

Next, we state the second lemma which we need for the proofs. We will use it to analyze the influence of the transfer function $\hat{h}_{T}$ on the approximation process.

Lemma 4. Let $\left\{t_{k}\right\}_{k \in \mathbb{Z}} \subset \mathbb{R}$ be an ordered complete interpolating sequence for $\mathcal{P W}_{\pi}^{2}$ and $\phi_{k}$ as defined in (6). For all $\omega \in[-\pi, \pi]$, all $t \in \mathbb{R}$, and all $N \in \mathbb{N}$ we have

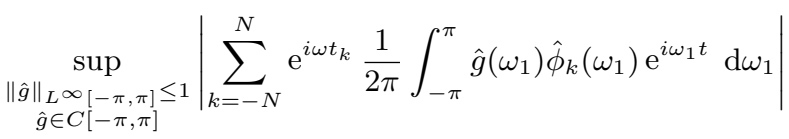

$$
\begin{aligned}
& =\frac{1}{2 \pi} \int_{-\pi}^{\pi}\left|\sum_{k=-N}^{N} \mathrm{e}^{i \omega t_{k}} \hat{\phi}_{k}\left(\omega_{1}\right)\right| \mathrm{d} \omega_{1} .
\end{aligned}
$$


Proof of Lemma 4. Let $\left\{t_{k}\right\}_{k \in \mathbb{Z}} \subset \mathbb{R}$ be an ordered complete interpolating sequence for $\mathcal{P} \mathcal{W}_{\pi}^{2}, \omega \in[-\pi, \pi], t \in \mathbb{R}$, and $N \in \mathbb{N}$, all be arbitrary but fixed. Further, let $\phi_{k}$ be defined as in (6). For

$$
\hat{g}\left(\omega_{1}\right)=\exp \left(-i \arg \left(\mathrm{e}^{i \omega_{1} t} \sum_{k=-N}^{N} \mathrm{e}^{i \omega t_{k}} \hat{\phi}_{k}\left(\omega_{1}\right)\right)\right)
$$

we have

$$
\begin{aligned}
& \left|\sum_{k=-N}^{N} \mathrm{e}^{i \omega t_{k}} \frac{1}{2 \pi} \int_{-\pi}^{\pi} \hat{g}\left(\omega_{1}\right) \hat{\phi}_{k}\left(\omega_{1}\right) \mathrm{e}^{i \omega_{1} t} \mathrm{~d} \omega_{1}\right| \\
& =\frac{1}{2 \pi} \int_{-\pi}^{\pi}\left|\sum_{k=-N}^{N} \mathrm{e}^{i \omega t_{k}} \hat{\phi}_{k}\left(\omega_{1}\right)\right| \mathrm{d} \omega_{1} .
\end{aligned}
$$

Further, as a consequence of Lusin's theorem [18, p. 56], there exists a sequence of functions $\left\{\hat{g}_{n}\right\}_{n \in \mathbb{N}}$ with $\hat{g}_{n} \in C[-\pi, \pi]$ and $\left\|\hat{g}_{n}\right\|_{L^{\infty}[-\pi, \pi]} \leq 1$, such that $\lim _{n \rightarrow \infty} \hat{g}_{n}\left(\omega_{1}\right)=\hat{g}\left(\omega_{1}\right)$ almost everywhere. It follows from Lebesgue's dominated convergence theorem and (16) that

$$
\begin{aligned}
\lim _{n \rightarrow \infty}\left|\sum_{k=-N}^{N} \mathrm{e}^{i \omega t_{k}} \frac{1}{2 \pi} \int_{-\pi}^{\pi} \hat{g}_{n}\left(\omega_{1}\right) \hat{\phi}_{k}\left(\omega_{1}\right) \mathrm{e}^{i \omega_{1} t} \mathrm{~d} \omega_{1}\right| \\
=\left|\sum_{k=-N}^{N} \mathrm{e}^{i \omega t_{k}} \frac{1}{2 \pi} \int_{-\pi}^{\pi} \hat{g}\left(\omega_{1}\right) \hat{\phi}_{k}\left(\omega_{1}\right) \mathrm{e}^{i \omega_{1} t} \mathrm{~d} \omega_{1}\right| \\
=\frac{1}{2 \pi} \int_{-\pi}^{\pi}\left|\sum_{k=-N}^{N} \mathrm{e}^{i \omega t_{k}} \hat{\phi}_{k}\left(\omega_{1}\right)\right| \mathrm{d} \omega_{1} .
\end{aligned}
$$

Hence, taking the limit $n \rightarrow \infty$ on both sides of

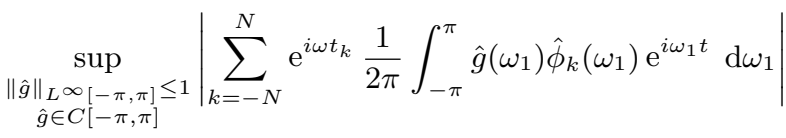

$$
\begin{aligned}
& \geq\left|\sum_{k=-N}^{N} \mathrm{e}^{i \omega t_{k}} \frac{1}{2 \pi} \int_{-\pi}^{\pi} \hat{g}_{n}\left(\omega_{1}\right) \hat{\phi}_{k}\left(\omega_{1}\right) \mathrm{e}^{i \omega_{1} t} \mathrm{~d} \omega_{1}\right|
\end{aligned}
$$

completes the proof.

Proof of Theorem 1. Let $\left\{t_{k}\right\}_{k \in \mathbb{Z}} \subset \mathbb{R}$ be an arbitrary but fixed ordered complete interpolating sequence for $\mathcal{P} \mathcal{W}_{\pi}^{2}$ and $\phi_{k}$ as defined in (6). Further, let $t \in \mathbb{R}$ be arbitrary but fixed.

From Lemma 1 we see that

$$
\sup _{N \in \mathbb{N}} \int_{-\pi}^{\pi}\left|\sum_{k=-N}^{N} \mathrm{e}^{i \omega t_{k}} \hat{\phi}_{k}\left(\omega_{1}\right)\right| \mathrm{d} \omega_{1}=\infty
$$

for all $\omega \in[-\pi, \pi]$. Due to Lemma 4 this implies that

$$
\begin{aligned}
\sup _{N \in \mathbb{N}}\left(\sup _{\substack{\left\|\hat{h}_{T}\right\|_{L} \infty[-\pi, \pi] \\
\hat{h}_{T} \in C[-\pi, \pi]}} \mid \sum_{k=-N}^{N} \mathrm{e}^{i \omega t_{k}} \times\right. \\
\left.\quad \times \frac{1}{2 \pi} \int_{-\pi}^{\pi} \hat{h}_{T}\left(\omega_{1}\right) \hat{\phi}_{k}\left(\omega_{1}\right) \mathrm{e}^{i \omega_{1} t} \mathrm{~d} \omega_{1} \mid\right)=\infty
\end{aligned}
$$

for all $\omega \in[-\pi, \pi]$. Thus, according to the Banach-Steinhaus theorem [18, p. 98], for all $\omega \in[-\pi, \pi]$ there exists a function $\hat{h}_{T_{\omega}} \in C[-\pi, \pi]$ such that

$$
\limsup _{N \rightarrow \infty}\left(\left|\sum_{k=-N}^{N} \mathrm{e}^{i \omega t_{k}} \frac{1}{2 \pi} \int_{-\pi}^{\pi} \hat{h}_{T_{\omega}}\left(\omega_{1}\right) \hat{\phi}_{k}\left(\omega_{1}\right) \mathrm{e}^{i \omega_{1} t} \mathrm{~d} \omega_{1}\right|\right)=\infty .
$$

Further, since $\hat{h}_{T_{\omega}} \in C[-\pi, \pi] \subset L^{\infty}[-\pi, \pi]$, and since there is the bijection (3) between $L^{\infty}[-\pi, \pi]$ and the set of stable LTI systems $T_{\omega}: \mathcal{P} \mathcal{W}_{\pi}^{1} \rightarrow \mathcal{P} \mathcal{W}_{\pi}^{1}$, it follows that for all $\omega \in[-\pi, \pi]$ there exists a stable LTI system $T_{\omega}$ such that

$$
\limsup _{N \rightarrow \infty}\left|\sum_{k=-N}^{N} \mathrm{e}^{i \omega t_{k}}\left(T_{\omega} \phi_{k}\right)(t)\right|=\infty .
$$

In particular, for $\omega=0$ there exists a stable LTI system $T_{*}=T_{0}$ such that

$$
\limsup _{N \rightarrow \infty}\left|\sum_{k=-N}^{N}\left(T_{*} \phi_{k}\right)(t)\right|=\infty
$$

$T_{0}$ is the desired stable LTI system $T_{*}$.

Next, let $0<\sigma<\pi$ be arbitrary but fixed. For $f \in \mathcal{P} \mathcal{W}_{\sigma}^{1}$ and $N \in \mathbb{N}$ we have

$$
\sum_{k=-N}^{N} f\left(t_{k}\right)\left(T_{*} \phi_{k}\right)(t)=\frac{1}{2 \pi} \int_{-\sigma}^{\sigma} \hat{f}\left(\omega_{1}\right) \sum_{k=-N}^{N} \mathrm{e}^{i \omega_{1} t_{k}}\left(T_{*} \phi_{k}\right)(t) \mathrm{d} \omega_{1} .
$$

Hence, it follows that

$$
\begin{aligned}
& \sup _{\|f\|_{\mathcal{P} \mathcal{W}_{\sigma}^{1}} \leq 1}\left|\sum_{k=-N}^{N} f\left(t_{k}\right)\left(T_{*} \phi_{k}\right)(t)\right| \\
& =\max _{\omega_{1} \in[-\sigma, \sigma]}\left|\sum_{k=-N}^{N} \mathrm{e}^{i \omega_{1} t_{k}}\left(T_{*} \phi_{k}\right)(t)\right| \geq\left|\sum_{k=-N}^{N}\left(T_{*} \phi_{k}\right)(t)\right| .
\end{aligned}
$$

Consequently, from (17) we obtain that

$$
\limsup _{N \rightarrow \infty}\left(\sup _{\|f\|_{\mathcal{P} \mathcal{W}_{\sigma}^{1} \leq 1}}\left|\sum_{k=-N}^{N} f\left(t_{k}\right)\left(T_{*} \phi_{k}\right)(t)\right|\right)=\infty .
$$

Thus, the Banach-Steinhaus theorem [18, p. 98] implies that there exists a signal $f_{*} \in \mathcal{P} \mathcal{W}_{\sigma}^{1}$ such that

$$
\limsup _{N \rightarrow \infty}\left|\sum_{k=-N}^{N} f_{*}\left(t_{k}\right)\left(T_{*} \phi_{k}\right)(t)\right|=\infty .
$$

Proof of Theorem 2. The proof of Theorem 2 is identical to the proof of Theorem 1, except that we choose $\omega \in\left[\sigma_{1}, \sigma_{2}\right]$ instead of $\omega=0$. Since the divergence creating stable LTI system $T_{*}$ depends on the actual choice of $\omega$, we see that $T_{*}$ is no longer universal in the sense that it is independent of $\sigma_{1}$ and $\sigma_{2}$.

\section{RELATION TO PRIOR WORK}

The approximation of LTI systems by sampling series has been studied for a long time $[19,20,21,22,23,8]$, but usually only for specific systems, e.g., the Hilbert transform or the differential operator $[21,8]$ or for the space $\mathcal{P} \mathcal{W}_{\pi}^{2}[19,20]$. In [23], Habib derives a sampling representation for certain bounded linear systems operating on the Zakai space. However, the class of systems for which this sampling representation holds is very restricted [24]. Sampling theorems for the signal space $\mathcal{P} \mathcal{W}_{\pi}^{1}$ were first studied in [25]. In this paper we study the system approximation problem for the space $\mathcal{P} \mathcal{W}_{\pi}^{1}$ and show that the approximation is not necessarily stable. This proves a conjecture from [10]. 


\section{REFERENCES}

[1] Claude E. Shannon, "Communication in the presence of noise," in Proceedings of the IRE, Jan. 1949, vol. 37, pp. 1021.

[2] Abdul J. Jerri, "The Shannon sampling theorem-its various extensions and applications: A tutorial review," Proc. IEEE, vol. 65, no. 11, pp. 1565-1596, Nov. 1977.

[3] John R. Higgins, "Five short stories about the cardinal series," Bull. Amer. Math. Soc., vol. 12, no. 1, pp. 45-89, 1985.

[4] Farokh Marvasti, Ed., Nonuniform Sampling: Theory and Practice, Kluwer Academic / Plenum Publishers, 2001.

[5] Richard P. Feynman, Feynman Lectures on Computation, Penguin Books, 1999.

[6] John R. Higgins, Sampling Theory in Fourier and Signal Analysis - Foundations, Oxford University Press, 1996.

[7] P. L. Butzer, W. Splettstößer, and R. L. Stens, "The sampling theorem and linear prediction in signal analysis," Jahresbericht der Deutschen Mathematiker-Vereinigung, vol. 90, no. 1, pp. 1-70, Jan. 1988.

[8] Paul L. Butzer, Gerhard Schmeisser, and Rudolf L. Stens, "Shannon's sampling theorem for bandlimited signals and their Hilbert transform, Boas-type formulae for higher order derivative- the aliasing error involved by their extensions from bandlimited to non-bandlimited signals," Entropy, vol. 14, no. 11, pp. 2192-2226, Nov. 2012.

[9] Holger Boche and Ullrich J. Mönich, "Sampling-type representations of signals and systems," Sampling Theory in Signal and Image Processing, vol. 9, no. 1-3, pp. 119-153, Jan., May, Sept. 2010.

[10] Holger Boche and Ullrich J. Mönich, "Sampling of deterministic signals and systems," IEEE Trans. Signal Process., vol. 59, no. 5, pp. 2101-2111, May 2011.

[11] Boris Yakovlevich Levin, Lectures on Entire Functions, AMS, 1996.

[12] Robert M. Young, An Introduction to Nonharmonic Fourier Series, Academic Press, 2001.
[13] Christopher Heil, A Basis Theory Primer: Expanded Edition, vol. 1 of Applied and Numerical Harmonic Analysis, Birkhäuser Boston, 2011.

[14] Stanislaw J. Szarek, "Nonexistence of Besselian basis in $C(S)$, , Journal of Functional Analysis, vol. 37, pp. 56-67, 1980.

[15] Paul L. Butzer, "The Hausdorff-Young theorems of Fourier analysis and their impact," Journal of Fourier Analysis and Applications, vol. 1, no. 2, pp. 113-130, May 1994.

[16] B. S. Pavlov, "Basicity of an exponential system and Muckenhoupt's condition," Dokl. Akad. Nauk SSSR, vol. 247, no. 1, pp. 37-40, 1979, English translation in Sov. Math. Dokl. 20 (1979), no. 4, 655-659.

[17] Ralph Philip Boas, Entire Functions, Academic Press, 1954.

[18] Walter Rudin, Real and Complex Analysis, McGraw-Hill, 3 edition, 1987.

[19] Henry P. Kramer, "The digital form of operators on bandlimited functions," Journal of Mathematical Analysis and Applications, vol. 44, no. 2, pp. 275-287, Nov. 1973.

[20] Athanasios Papoulis, "Generalized sampling expansion," IEEE Trans. Circuits Syst., vol. 24, no. 11, pp. 652-654, Nov. 1977.

[21] Rudolf L. Stens, "A unified approach to sampling theorems for derivatives and Hilbert transforms," Signal Processing, vol. 5, pp. 139-151, 1983.

[22] Alan V. Oppenheim and Ronald W. Schafer, Discrete-Time Signal Processing, Prentice Hall, 3 edition, 2009.

[23] Muhammad K. Habib, "Digital representations of operators on band-limited random signals," IEEE Trans. Inf. Theory, vol. 47, no. 1, pp. 173-177, Jan. 2001.

[24] Holger Boche and Ullrich J. Mönich, "System representations for the Zakai class with applications," IEEE Trans. Inf. Theory, vol. 56, no. 8, pp. 4129-4134, Aug. 2010.

[25] J. L. Brown, Jr., "Bounds for truncation error in sampling expansions of band-limited signals," IEEE Trans. Inf. Theory, vol. 15, no. 4, pp. 440-444, July 1969. 